\title{
Interferometric Technique for the Spectral Characterization of High Frequency Current-Modulated Mid-Infrared Semiconductor Lasers
}

\author{
Marc-Simon Bahr ${ }^{1,2, *}$ and Marcus Wolff ${ }^{1}$ (D) \\ 1 Heinrich Blasius Institute of Physical Technologies, Hamburg University of Applied Sciences, Berliner Tor 21, \\ 20099 Hamburg, Germany; marcus.wolff@haw-hamburg.de \\ 2 School of Computing, Engineering and Physical Sciences, University of the West of Scotland, High Street, \\ Paisley PA1 2BE, UK \\ * Correspondence: marc-simon.bahr@haw-hamburg.de
}

check for

updates

Citation: Bahr, M.-S.; Wolff, M Interferometric Technique for the Spectral Characterization of High Frequency Current-Modulated Mid-Infrared Semiconductor Lasers. Photonics 2021, 8, 443. https:// doi.org/10.3390/photonics 8100443

Received: 19 August 2021

Accepted: 9 October 2021

Published: 14 October 2021

Publisher's Note: MDPI stays neutral with regard to jurisdictional claims in published maps and institutional affiliations.

Copyright: (c) 2021 by the authors. Licensee MDPI, Basel, Switzerland. This article is an open access article distributed under the terms and conditions of the Creative Commons Attribution (CC BY) license (https:/ / creativecommons.org/licenses/by/ $4.0 /)$.

\begin{abstract}
We describe two methods, based on Michelson interferometery, that enable the determination of the absolute wavelength of current-modulated semiconductor lasers. By non-linear regression of the instantaneous frequency of the interference signal, the rate of change of the wavelength of the radiation can be inferred. Alternatively, the absolute wavelength can be directly calculated from the maxima and minima of the interference signal. In both cases a reference absorption line enables the determination of the absolute wavelength. Both methods offer respective advantages. The methods allow a highly resolved wavelength measurement under lower kilohertz range current-modulation with relatively little effort. As a result, we present the rates of wavelength change and absolute wavelengths exemplarily for a specific interband cascade laser. It is furthermore shown that the spectral dynamic range of the laser decreases with increasing modulation frequency.
\end{abstract}

Keywords: current modulation; interband cascade laser; michelson interferometer; high-resolution spectroscopy

\section{Introduction}

The exact knowledge of the emission wavelength is one of the fundamental parameters in laser spectroscopy. Several spectroscopic techniques such as photoacoustics require a modulation of the radiation as a matter of principle [1].

Semiconductor lasers can easily be modulated by variation of the operating current. In doing so amplitude as well as wavelength modulation are possible. However, assuming a simple, frequency-independent proportionality between wavelength and current, as it is observable in continuous wave $(\mathrm{cw})$ operation, can lead to significant measurement errors [2]. The determination of the momentary wavelength becomes extraordinarily difficult if the semiconductor laser emitting in the mid-infrared (e.g., an interband cascade laser or ICL) is modulated with a higher frequency.

At present, different methods and measuring devices exist that enable the spectral characterization of semiconductor lasers such as ICLs [3]. However, all of them are primarily applicable for $\mathrm{cw}$ radiation. Often, prism or grating spectrometers are used as measuring instruments, which are based on diffraction and interference phenomena, respectively. A further method is the Fourier transform interferometer (FTIR), which is based on a Michelson interferometer and determines the emission spectrum by Fourier transform of the interferogram [4].

Another possibility is the "wavemeter" of BRISTOL Instruments, Inc. (Victor, New York, NY, USA). It measures the wavelength of a laser on the basis of a Michelson interferometer, provides accuracy in the ppm range and represents the state of the art for wavelength measurements in continuous wave operation [5-7]. 
If the laser is operated in current-modulation mode, there are only few possibilities. One approach determines the wavelength change as a function of the laser operating current $(d \lambda / d I)$ [8]. The experimental setup is based on a Mach-Zehnder interferometer and the change in wavelength is calculated using a mathematical algorithm which is not further described. This method does not allow a determination of the absolute wavelength as a function of the current.

Another possibility for a fast wavelength measurement is a heterodyne (or superheterodyne) Michelson interferometer. It differs from the homodyne interferometer in that a beat signal is analyzed that results from the superposition of the radiation to be analyzed and that of a second laser. This technique is particularly well suited if the phase of the beat signal is required [9-11].

A US patent from 2019 represents the latest research progress regarding the determination of the absolute wavelength of current-modulated semiconductor lasers. It is pointed out that, on the basis of an interferometer, the absolute wavelength of tunable lasers can be determined very precisely. The rudimentarily described method mentions the need for a reference wavelength, e.g., a gas absorption line [12].

To the best of our knowledge there is only one commercially available device for the determination of mid-infrared wavelengths in modulation mode. The "NLIR 2.0-5.0 $\mu \mathrm{m}$ spectrometer" of NLIR ApS (Farum, Denmark) is based on non-linear optics and transforms the laser radiation into the near-infrared range, which is subsequent analyzed using a diffraction grating and a CMOS sensor [13]. According to the manufacturer the maximum sampling rate of the instrument is $130 \mathrm{kHz}$. The spectral resolution of the NLIR is around $1.5 \mathrm{~nm}$.

Due to the fact that the currently available methods for the spectral characterization of current-modulated semiconductor lasers, especially DFB interband cascade lasers, are not sufficient to achieve a high spectral resolution, new techniques are required.

\section{Materials and Methods}

In the following, two methods for determining the absolute wavelength of modulated semiconductor lasers, in this case interband cascade lasers, are described. Both are based on the same experimental setup and exhibit advantages and disadvantages due to the different signal processing.

\subsection{Experimental Setup}

Figure 1 shows the schematic experimental setup for measuring the laser wavelength as function of the current during modulation. It is based on a homodyne Michelson interferometer with silver mirrors and $\mathrm{a} \mathrm{CaF}_{2}$ beam splitter [14]. The difference between the two distances $a$ and $b$ is of great importance, because it determines the quality of the results. If $a$ and $b$ approach each other, the number of maxima and minima in the interference signal decreases, which is disadvantageous for the spectral characterization. Simultaneously, the signal-to-noise ratio (SNR) increases due to the decreasing distance to the detector. This has a positive effect on the evaluation. If one of the two paths becomes significantly larger than the other, the number of maxima and minima increases, however, the SNR decreases due to the longer distance to the detector. In order to achieve a good compromise between spectral resolution and SNR, the distances have to be empirically optimized.

The Thorlabs PDA07P2 employs an InAsSb detector and provides a bandwidth of $9 \mathrm{MHz}$. The ICL 1541, manufactured by nanoplus GmbH (Gerbrunn, Germany), was characterized with an average wavelength $\lambda_{0}$ of $3333 \mathrm{~nm}$ and a spectral linewidth of less than $20 \mathrm{MHz}$. The laser can be operated at temperatures between 15 and 27 degrees Celsius. Stimulated emission occurs with operating currents between 25 and $45 \mathrm{~mA}$. The average optical output power is $5.5 \mathrm{~mW}$. During $\mathrm{cw}$ operation it can be assumed that the wavelength is proportional to the current with $d \lambda / d I=0.16 \mathrm{~nm} / \mathrm{mA}$. The ICL is driven by the Thorlabs TLD001 diode driver and its temperature is controlled by the Thorlabs temperature controller TTC001. The laser diode current can be accurately set to $10 \mu \mathrm{A}$ with 
a noise level $<3 \mu \mathrm{A}$ rms. The ICL chip is installed in a TO66 housing including a Peltier element for temperature regulation and the temperature sensor. Within the scope of the technical possibilities, the laser wavelength is optimally stabilized. A 33220A function generator (Agilent, Santa Clara, CA, USA) is used to modulate the operating current of the diode driver.

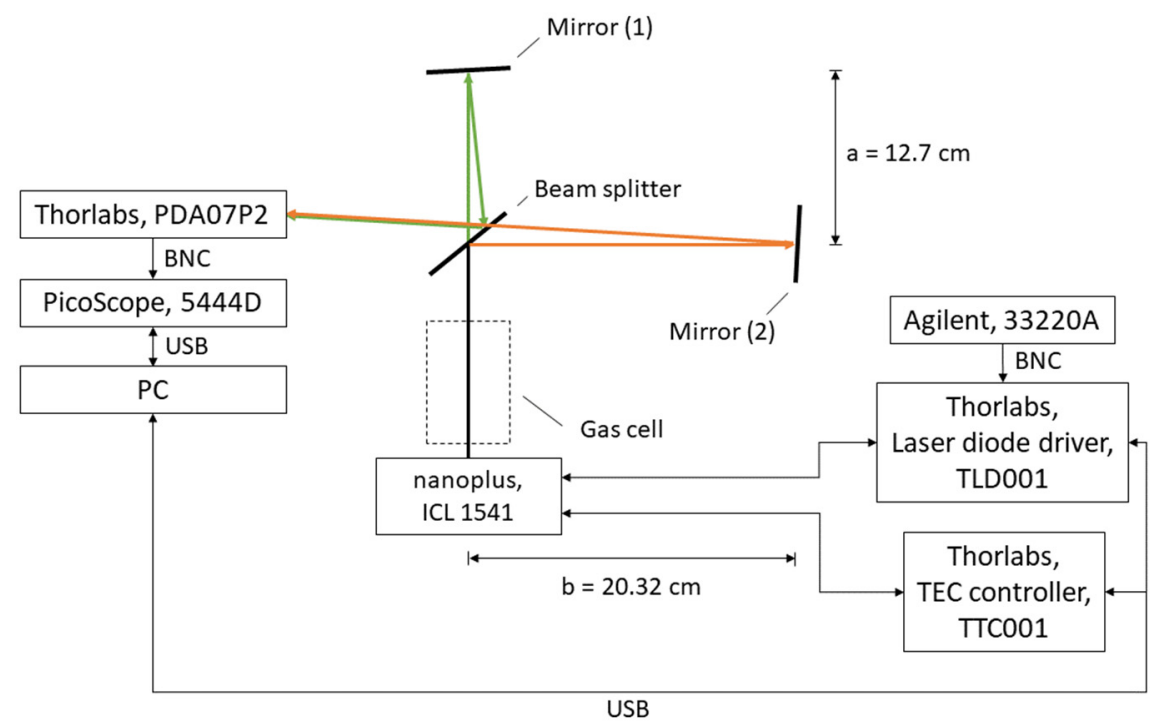

Figure 1. Experimental setup based on a Michelson interferometer.

The intensity detected by the detector is converted into a voltage signal which is sampled by a PicoScope 5444D (Pico Technology, Cambridgeshire, UK) with 16 bit resolution. The sampling rate is $62.5 \mathrm{MS} / \mathrm{s}$. The sampled signal is then subjected to processing by means of MATLAB. After the signal is time sliced, Savitzky-Golay based filtering of the signal is performed to eliminate high frequency noise [15]. This optimizes the precision of the subsequent detection of the local maxima and minima in the interference signal. Subsequently, different mathematical equations and methods are applied according to the procedures described in Sections 2.2 and 2.3, respectively.

\subsection{Non-Linear Regression}

\subsubsection{Spectral Characterization}

The electric field (E-field) component of the ICL radiation at the location of the detector can be described as [16]:

$$
E(t)=\hat{E}(t) \cdot e^{j\left(\omega t+\varphi_{0}\right)}
$$

where $\omega$ is the instantaneous angular frequency of the E-field and $\varphi_{0}$ is the initial phase of the laser radiation. The stochastic phase noise of the laser radiation is neglected here, since it would only increase the complexity of the equation and does not add any value to the following calculations. $\hat{E}(t)$ corresponds to the amplitude and is time-dependent because the ICL is modulated.

One part of the laser beam is transmitted through the beam splitter and reflected by mirror (1). When it hits the detector, its E-Field equals:

$$
E_{1}(t)=\hat{E}(t) \cdot e^{j\left(\omega t+\varphi_{0}\right)} .
$$

The second part of the laser beam is reflected at the beam splitter, then reflected at mirror (2) and subsequently transmitted through the beam splitter. Due to the path difference $s_{d}=2(b-a)$, it hits the detector delayed by a time $t_{d}$ compared to $E_{1}(t)$ :

$$
t_{d}=\frac{2(b-a)}{c}=\frac{s_{d}}{c}
$$


where $c$ corresponds to the speed of light. If this part of the laser beam hits the detector, the E-field component can be described with:

$$
E_{2}(t)=E_{1}\left(t-t_{d}\right)=\hat{E}\left(t-t_{d}\right) \cdot e^{j\left(\omega\left(t-t_{d}\right)+\varphi_{0}\right)} .
$$

If the laser current is modulated, the wavelength (angular frequency $\omega$ ) changes continuously as a function of time. This results in the following relationships for the E-field components hitting the detector:

$$
\begin{gathered}
E_{1}(t)=\hat{E}(t) \cdot e^{j\left(\int_{0}^{t} \omega(\tau) d \tau+\varphi_{0}\right)}, \\
E_{2}(t)=\hat{E}\left(t-t_{d}\right) \cdot e^{j\left(\int_{0}^{t-t} \omega(\tau) d \tau+\varphi_{0}\right)} .
\end{gathered}
$$

The integration of the angular frequency becomes necessary because it is not constant over time anymore.

If both partial beams hit the detector, the sum of both components equals:

$$
E_{S}(t)=E_{1}(t)+E_{2}(t)
$$

Since the detector only detects intensities, the relationship between the E-field components and the intensity must be applied [17]:

$$
I(t) \propto\left|E_{s}(t)\right|^{2} .
$$

Since the absolute value of the intensity is of no importance for the further considerations, the following results for the detector signal:

$$
I(t) \propto \hat{E}^{2}(t)+\hat{E}^{2}\left(t-t_{d}\right)+2 \hat{E}(t) \cdot \hat{E}\left(t-t_{d}\right) \cdot \cos \left(\int_{t-t_{d}}^{t} \omega(\tau) d \tau\right) .
$$

Due to the extremely short interferometer delay time $\left(t_{d}<1 \mathrm{~ns}\right)$, it can be assumed that $E(t) \approx E\left(t-t_{d}\right)$, which simplifies Equation (9) to:

$$
I(t) \propto 2 \hat{E}^{2}(t)+2 \hat{E}^{2}(t) \cdot \cos \left(\int_{t-t_{d}}^{t} \omega(\tau) d \tau\right) .
$$

Therefore, the detector signal depends particularly on the interferometer delay time and corresponds theoretically to an oscillation between $4 \hat{E}^{2}(t)$ and zero. The instantaneous angular frequency of the laser $\omega(t)$ can be derived from the phase $\varphi(t)$ of the detector signal. This is possible due to the elementary relationship between the angular frequency and the phase $\omega(t)=\frac{d \varphi(t)}{d t}[18]$ :

$$
\omega_{I f}(t)=\dot{\varphi}(t)=\frac{d}{d t}\left(\int_{t-t_{d}}^{t} \omega(\tau) d \tau\right),
$$

where $\omega_{I f}(t)$ corresponds to the angular frequency of the interferometer signal. Since the integration interval in Equation (11) corresponds to the delay time $t_{d}$, the infinitesimal value $\omega(\tau)$ can be considered approximately constant in the interval. Thus Equation (11) results in:

$$
\omega_{I f}(t)=\frac{d}{d t} \omega(t) \cdot t_{d}=2 \pi \cdot c \cdot t_{d} \cdot \frac{d}{d t} \frac{1}{\lambda(t)}=-\frac{2 \pi \cdot c \cdot t_{d}}{\lambda^{2}(t)} \cdot \frac{d \lambda(t)}{d t} .
$$

The square of the wavelength $\lambda^{2}(t)$ can be approximated by the square of the average wavelength $\lambda_{0}^{2}$ given in the data sheet of the respective semiconductor laser. Understandably, this does not apply to $\lambda(t)$ in the derivative. Resolved according to the wavelength 
change as function of time and with the relationship $s_{d}=c \cdot t_{d}$ according to Equation (3), it follows that:

$$
\frac{d \lambda(t)}{d t}=-\frac{f_{I f}(t) \cdot \lambda_{0}^{2}}{c \cdot t_{d}}=-\frac{f_{I f}(t) \cdot \lambda_{0}^{2}}{s_{d}},
$$

with the momentary frequency of the interference $f_{I f}(t)$. The negative sign on the right side has no meaning for further calculations. Due to the symmetry of the cosine function, it is not possible to distinguish between positive and negative phases of the interferometer signal. The negative sign can therefore be omitted.

However, the wavelength change as function of the change in current is sought. The modulation of the current is, in this case, sawtooth-shaped with the rate of change $k$ (units: $\frac{\mathrm{mA}}{\mathrm{s}}$ ):

$$
\frac{d I(t)}{d t}=k
$$

Equation (14) solved for $d t$ and inserted into Equation (13) delivers (if the sign is omitted):

$$
\frac{d \lambda(I)}{d I}=\frac{f_{I f}(I) \cdot \lambda_{0}^{2}}{s_{d} \cdot k}
$$

The wavelength as a function of the operating current can be determined from the differential quotient by means of integration if an absolute wavelength is known as reference point:

$$
\lambda\left(I_{1}\right)=\int_{I_{0}}^{I_{1}} \frac{d \lambda(I)}{d I} d I+\lambda\left(I_{0}\right) .
$$

The reference wavelength $\lambda\left(I_{0}\right)$ can, for instance, be determined experimentally. If the modulated laser beam passes a cell filled with a gas that shows a strong and distinct absorption line in the expected wavelength range, the transmission of the interference signal provides the reference wavelength [19].

\subsubsection{Error Estimation}

The wavelength as function of current calculated according to Equation (16) is subject to deviations due to measurement errors and approximations.

Equation (15) inserted into Equation (16) results in:

$$
\lambda\left(I_{1}\right)=\frac{\lambda_{0}^{2}}{s_{d} \cdot k} \int_{I_{0}}^{I_{1}} f(I) d I+\lambda\left(I_{0}\right) .
$$

The coefficients of the regression function $f(I)$ can be determined applying a polynomial regression of second (or larger) degree using the evaluated frequency values of the interference signal. After integration, a closed analytical expression is obtained, depending on metrologically determined quantities, which are subject to error tolerances. The maximum possible error can then be calculated by the sum of the following components:

$$
\begin{gathered}
\Delta \lambda\left(I_{1}\right)_{\lambda_{0}}=\frac{2 \lambda_{0}}{s_{d} \cdot k}\left(\frac{a_{1}}{3} I_{1}^{3}+\frac{a_{2}}{2} I_{1}^{2}+a_{3} I_{1}-\frac{a_{1}}{3} I_{0}^{3}-\frac{a_{2}}{2} I_{0}^{2}-a_{3} I_{0}\right) \cdot \Delta \lambda_{0}, \\
\Delta \lambda\left(I_{1}\right)_{s_{d}}=\frac{\lambda_{0}^{2}}{s_{d}^{2} \cdot k}\left(\frac{a_{1}}{3} I_{1}^{3}+\frac{a_{2}}{2} I_{1}^{2}+a_{3} I_{1}-\frac{a_{1}}{3} I_{0}^{3}-\frac{a_{2}}{2} I_{0}^{2}-a_{3} I_{0}\right) \cdot \Delta s_{d} \text { and } \\
\Delta \lambda\left(I_{1}\right)_{\lambda\left(I_{0}\right)}=\Delta \lambda\left(I_{0}\right),
\end{gathered}
$$

where $a_{1 \ldots 3}$ stand for the coefficients of the regression function and $\Delta \lambda_{0}, \Delta s_{d}$ and $\Delta \lambda\left(I_{0}\right)$ for the absolute errors of the individual variables. The influence of the integration according to Equation (17) on the maximum error can be estimated by the Riemann sum [20]. It includes the standard deviation of the instantaneous frequency $f(I)$, which results from the non-linear regression. 
An additional error is caused by approximations. One of the effects is the standard deviation $\sigma_{I f}$ of the interference signal which describes the distribution of the instantaneous frequencies around the regression function. For its share of the maximum wavelength error $\Delta \lambda_{\sigma I f}$ applies:

$$
\Delta \lambda_{\sigma I f}=\frac{\lambda_{0}^{2}}{s_{d} \cdot k} \cdot \pm \sigma_{I f} \cdot\left(I_{1}-I_{0}\right) .
$$

The result must be added to that from Equations (18)-(20).

\subsection{Direct Evaluation of Fringes}

\subsubsection{Spectral Characterization}

The equations presented in Section 2.2 are also valid for the method labeled "direct evaluation of fringes". The cosine occurring in the interference signal, according to Equation (10), corresponds to local maxima and minima, hereafter referred to as fringes, due to constructive and destructive interference.

The conditions for constructive and destructive interference depend mainly on the path difference of the Michelson interferometer whereas:

$$
\begin{gathered}
s_{d}=n \cdot \lambda, \\
s_{d}=(2 n+1) \cdot \frac{\lambda}{2},
\end{gathered}
$$

respectively, with $n \in \mathbb{Z}$ [21]. By means of a reference wavelength based on an absorption line in the transmission spectrum, the absolute wavelengths of the individual fringes according to Equations (22) and (23) can be calculated: After $n$ of the local extremum nearest to the reference wavelength has been determined, all other extrema can be assigned by iterating $n$. Subsequently, the wavelengths in between the individual extreme points are calculated by non-linear regression.

\subsubsection{Error Estimation}

The accuracy of the results of this method depends, besides the exactness of the reference wavelength, mainly on the accuracy of the path difference $s_{d}$. Its influence on the maximum error of the frequency of fringes $f_{f}$ results in:

$$
\begin{gathered}
\Delta f_{f}=n \cdot \frac{c}{s_{d}^{2}} \cdot \Delta s_{d}, \\
\Delta f_{f}=\frac{2 n+1}{2} \cdot \frac{c}{s_{d}^{2}} \cdot \Delta s_{d},
\end{gathered}
$$

for constructive and destructive interference, respectively, where $\Delta s_{d}$ corresponds to the maximum error of $s_{d}$ and the natural number $n$ to the number of fringes which lie to the left or right of the reference wavelength. Due to the linear relationship between $\Delta f_{f}$ and $n$, it is advantageous to base the calculation on the frequency and convert it subsequently to wavelength.

\section{Results}

The two methods were tested using the nanoplus ICL 1541. For the test a sawtooth modulation between 25 and $45 \mathrm{~mA}$ was applied. Measurements were conducted at 15 , 21 and $27^{\circ} \mathrm{C}$ in order to cover the entire dynamic range. The gas used for the determination of the reference wavelength is ethane with a purity of $99.999 \%$ and $99 \%$ nitrogen content. Measurements were taken at a gas temperature of approximately $22{ }^{\circ} \mathrm{C}$ and a pressure of 1000 mbar.

Figure 2 shows exemplary results obtained by the two methods. 


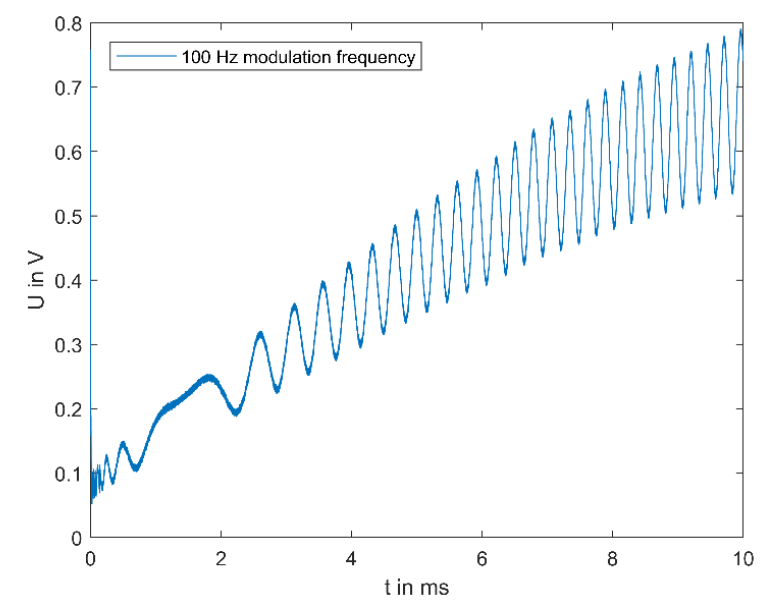

(a)

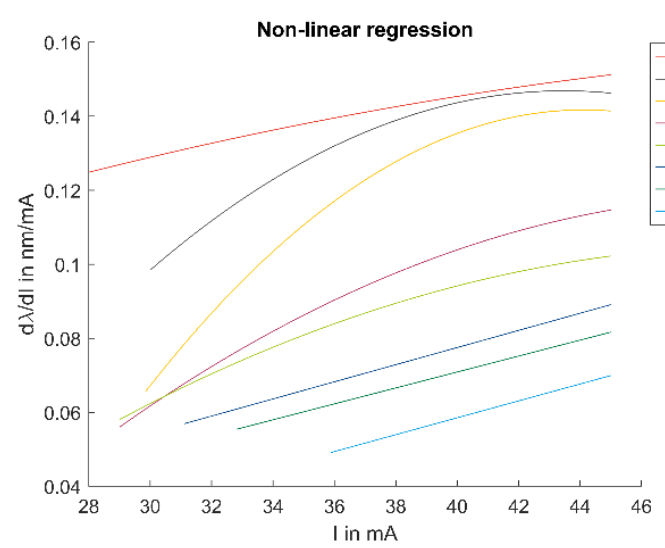

(b)

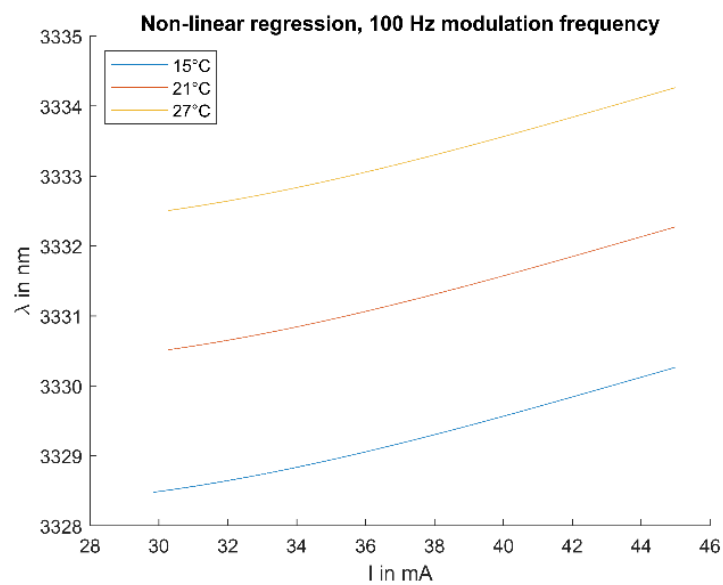

(d)

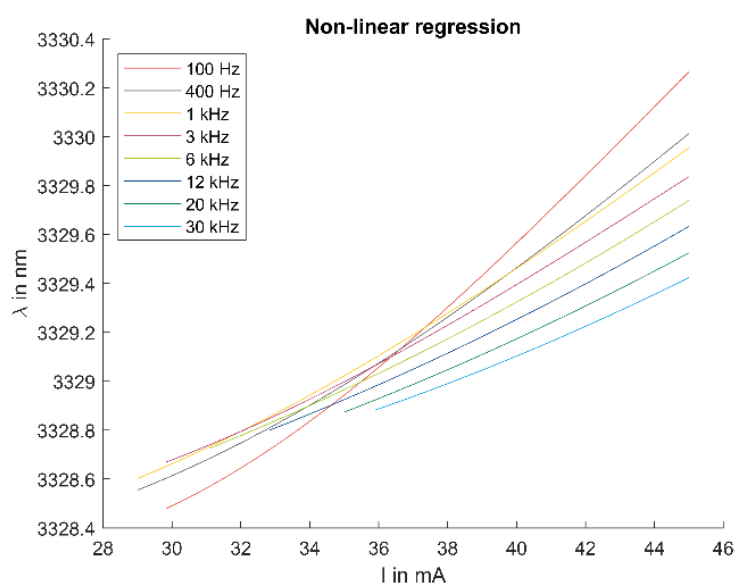

(c)

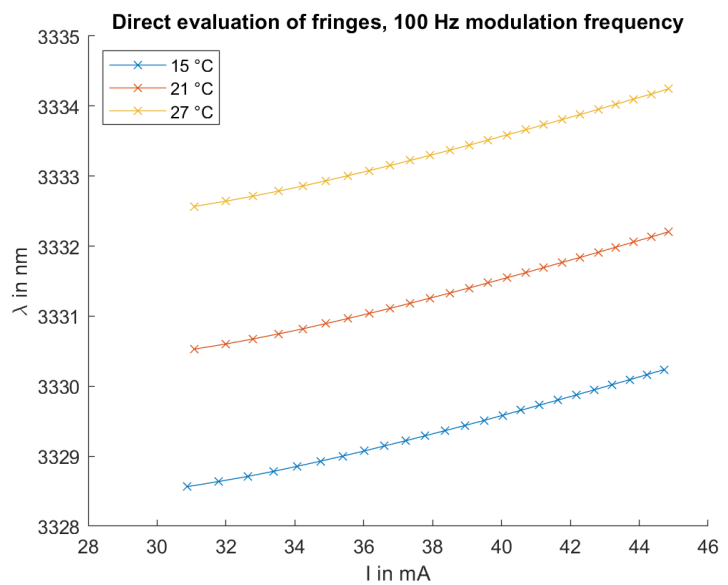

(e)

Figure 2. Measurements with the nanoplus ICL 1541 at sawtooth modulation: (a) Exemplary interference signal at $100 \mathrm{~Hz}$ modulation frequency and $15{ }^{\circ} \mathrm{C}$ laser temperature; (b) Change of wavelength as function of operating current for different modulation frequencies based on the non-linear regression method at $15^{\circ} \mathrm{C}$ laser temperature; (c) Absolute wavelength as function of operating current for different modulation frequencies based on the non-linear regression method at $15^{\circ} \mathrm{C}$ laser temperature; (d) Absolute wavelength as function of the operating current for three different laser temperatures based on the non-linear regression method at $100 \mathrm{~Hz}$ modulation frequency; (e) Absolute wavelength as function of the operating current for three different laser temperatures based on the direct evaluation of fringes at $100 \mathrm{~Hz}$ modulation frequency; the crosses mark the calculation points. 
The measuring ranges of the two methods differ, because discontinuities in the interference signal are bridged in the non-linear regression method. They can be extracted directly from Figure $2 \mathrm{~d}$ and e, respectively. Table 1 lists the measuring ranges as well as the wavelength errors according to Equations (18)-(21).

Table 1. Measuring ranges and wavelength errors of the two methods.

\begin{tabular}{ccc}
\hline Method & Measuring Range & Wavelength Error \\
\hline Non-linear Regression method & $30-45 \mathrm{~mA}$ & $\Delta \lambda= \pm 0.12 \mathrm{~nm}$ \\
Direct evaluation of fringes & $31-45 \mathrm{~mA}$ & $\Delta \lambda= \pm 0.030 \mathrm{~nm}$ \\
\hline
\end{tabular}

Table 2 presents the spectral range of the ICL as function of the modulation frequency.

Table 2. Spectral range of nanoplus ICL 1541.

\begin{tabular}{cccc}
\hline Modulation Frequency $\mathbf{( k H z})$ & Wavelength Range $(\mathbf{n m})$ & $\boldsymbol{\Delta} \boldsymbol{\lambda}(\mathbf{n m})$ & $\boldsymbol{\Delta} \boldsymbol{\lambda}$ (Relative to cw) \\
\hline cW & $3327.52-3330.57$ & 3.05 & 1.00 \\
0.1 & $3328.48-3330.27$ & 1.79 & 0.59 \\
0.4 & $3328.55-3330.01$ & 1.46 & 0.48 \\
1 & $3328.60-3329.95$ & 1.35 & 0.44 \\
3 & $3328.67-3329.84$ & 1.17 & 0.38 \\
6 & $3328.73-3329.74$ & 1.01 & 0.33 \\
12 & $3328.80-3329.63$ & 0.83 & 0.27 \\
20 & $3328.87-3329.53$ & 0.66 & 0.22 \\
30 & $3328.88-3329.42$ & 0.54 & 0.18 \\
\hline
\end{tabular}

Figure 3 shows the spectral dynamic range $\Delta \lambda$ (relative to cw) as a function of frequency on the basis of the values in Table 2. According to the shape of the graph in Figure 3 , it is reasonable to assume that the relationship shown corresponds to the natural logarithm.

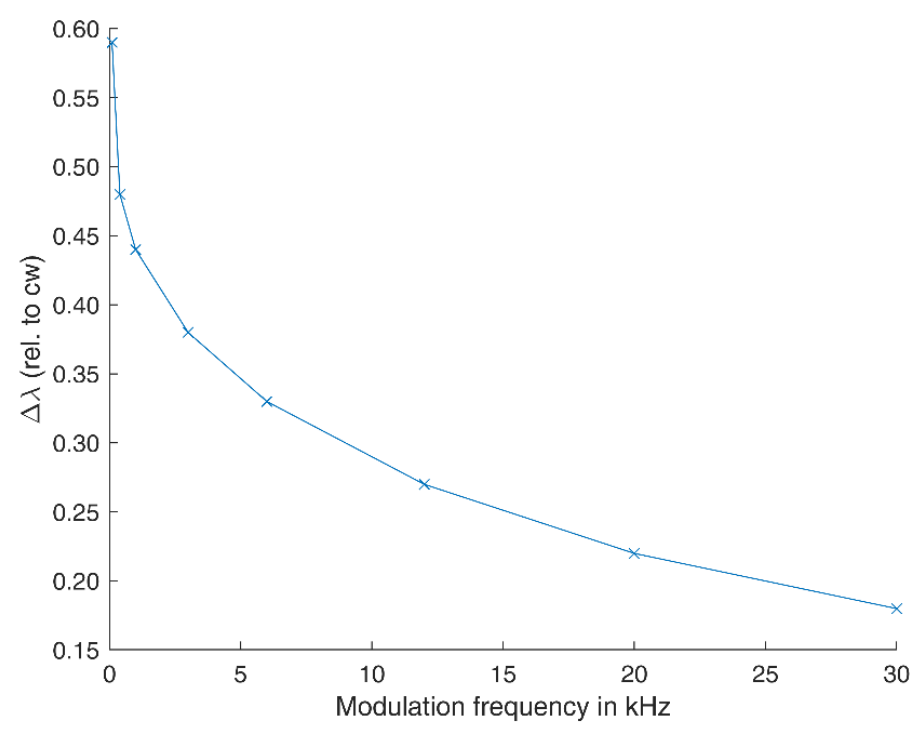

Figure 3. Spectral dynamic range $\Delta \lambda$ (relative to $\mathrm{cw}$ ) as function of modulation frequency.

\section{Discussion}

\subsection{Advantages and Disadvantages of Both Methods}

A direct comparison of the methods in Section 2 shows that the direct evaluation of fringes requires fewer physical quantities for the calculation of the absolute wavelength and less computational effort compared to the non-linear regression method. This results in an overall higher precision. 
The "non-linear regression method" on the other hand enables a compensation of discontinuities in the interference signal and, therefore, a larger measuring range compared to the direct evaluation of the fringes.

\subsection{Explanatory Approach}

Due to the lack of spectrometric tools, the spectral performance of mid-infrared semiconductor lasers under modulation has not yet been systematically investigated. The fact that the spectral dynamic range (i.e., the spectral modulation depth) decreases with increasing modulation frequency, as shown in Table 2 and Figure 3, was new even to the laser manufacturer. There is also no theoretical approach with which these measurement results could be compared.

However, the emission wavelength of a semiconductor laser is primarily determined by the geometric dimensions and the refractive index of the semiconductor material, and both parameters are significantly influenced by the laser temperature and thus indirectly by the injection current. A plausible explanation could therefore be a frequency-dependent temperature distribution of the semiconductor laser material. As the modulation frequency increases, there is less time available for establishing a thermal equilibrium. A changing frequency would therefore lead to a changing temperature distribution or the occurrence of (additional) inhomogeneities. Effects on the spectral emission are to be expected [22].

\section{Conclusions}

Figure 2a does not perfectly correspond to the mathematical description given by Equation (10). From the equation it would be expected that mutual cancellation of the DC and AC components and adding occur alternately. However, this is not observable because on the one hand Equation (10) is based on several approximations and on the other hand the alignment of the individual partial beams in the Michelson interferometer is not perfect.

An analysis of the results presented in Figure 2d,e shows that the wavelength difference between the two methods is only of the order of a few hundredths of a nanometer. Figure $2 b, c$ demonstrate that, with increasing modulation frequency, the wavelength change as function of operating current decreases. This limits the spectral range of the ICL which is particularly relevant for wavelength modulation.

The spectral tuning characteristics of the modulated laser $d \lambda(I) / d I$ (Figure $2 b$ ) and $\lambda(I)$ (Figure 2c-e) can be approximated by quadratic polynomials and cubic polynomials, respectively. Compared to $\mathrm{cw}$ operation, the ICL thus shows a clearly nonlinear spectral behavior.

In cw operation, the tuning coefficient $d \lambda / d I$ for the nanoplus ICL 1541 equals approximately $0.16 \frac{\mathrm{nm}}{\mathrm{mA}}$. Figure $2 \mathrm{~b}$ shows that with decreasing modulation frequencies the individual curves approach this constant value.

The direct evaluation of fringes is a factor of four more precise than the non-linear regression method, as fewer error-prone variables are included. However, the accuracy of both methods strongly depends on the precision of the reference wavelength derived from the transmission spectrum. An advantage of the non-linear regression method over the direct evaluation of fringes is a larger measurement range.

Compared to the state of art, both methods achieve a significantly higher resolution, which does additionally have the potential to be optimized by adjusting the path length difference of the Michelson interferometer.

A heterodyne (or super-heterodyne) interferometer would not provide advantages over the applied homodyne setup, especially with regard to spectral resolution. A significant disadvantage represents the need of a second often frequency-stabilized laser.

In conclusion, it should be mentioned that both methods can be adapted to other types of modulation (e.g., sinusoidal) and applied for the analysis of spectra recorded by wavelength modulation spectroscopy [23]. 
Author Contributions: Writing—original draft preparations: M.-S.B.; writing—review and editing: M.W. Both authors have read and agreed to the published version of the manuscript.

Funding: This research was funded by Federal Ministry for the Environment, Nature Conservation and Nuclear Safety, grant number 67KI2075.

Data Availability Statement: Not applicable.

Acknowledgments: We would like to thank Alain Loh. He gave the initial suggestion to measure the wavelength by means of a Michelson interferometer.

Conflicts of Interest: The authors declare no conflict of interest.

\section{References}

1. Besson, J.-P.; Schilt, S.; Thévenaz, L. Multi-gas sensing based on photoacoustic spectroscopy using tunable laser diodes. Spectrochim. Acta Part A Mol. Biomol. Spectrosc. 2005, 60, 3449-3456. [CrossRef] [PubMed]

2. $\quad$ Demtröder, W. Laserspektroskopie: Grundlagen und Techniken; Springer: Berlin/Heidelberg, Germany, 2011.

3. Meyer, J.R.; Bewley, W.W.; Canedy, C.L.; Kim, C.S.; Kim, M.K.C.D.; Merritt, C.D.; Vurgaftman, I. The Interband Cascade Laser. Photonics 2020, 7, 75. [CrossRef]

4. Smith, B.C. Fundamentals of Fourier Transform Infrared Spectroscopy; CRC Press: Boca Raton, FL, USA, 2011. [CrossRef]

5. Available online: https://www.bristol-inst.com/bristol-instruments-products/wavelength-meters-scientific (accessed on 14 August 2021).

6. Bristol Instruments, Inc. Optical Wavelength Meter 828 Series Datasheet. 2020. Available online: https://www.bristol-inst.com/ wp-content/uploads/2020/01/Bristol-828-Series-Optical-Wavelength-Meter-Specifications.pdf (accessed on 22 September 2021).

7. Veracious Statistics Research. Global Laser Wavelength Meters Research Report 2021; Professional Edition; Veracious Statistics Research: New Delhi, India, 2021. Available online: https:/ /vstatisticsresearch.com/global-laser-wavelength-meters-market (accessed on 22 September 2021).

8. Du, Z.; Luo, G.; An, Y.; Li, J. Dynamic spectral characteristics measurement of DFB interband cascade laser under injection current tuning. Appl. Phys. Lett. 2016, 109, 011903. [CrossRef]

9. Schuldt, T.; Kraus, H.-J.; Weise, D.; Braxmaier, C.; Peters, A.; Johann, U. A heterodyne interferometer for high resolution translation and tilt measurement as optical readout for the LISA inertial sensor. In Proceedings of the International Conference on Space Optics 2006-CSO 2006, Noordwijk, The Netherlands, 27-30 June 2006. [CrossRef]

10. Olyaee, S.; Yoon, T.; Hamedi, S. Jones matrix analysis of frequency mixing error in three-longitudinal-mode laser heterodyne interferometer. IET Optoelectron. 2009, 3, 215-224. [CrossRef]

11. Dändliker, R.; Thalmann, R.; Prongué, D. Two-wavelength laser interferometry using superheterodyne detection. Opt. Lett. 1988, 13, 339-341. [CrossRef] [PubMed]

12. Seeley, R.; Froggatt, M.E. High Precision Wavelength Measurement and Control of a Tunable Laser. U.S. Patent 10,502,632 B2, 10 December 2019.

13. Available online: https://nlir.com/2-0-5-0-\%c2\%b5m-spectrometer (accessed on 14 August 2021).

14. Steel, W.H. Interferometry; Cambridge University Press: Cambridge, UK, 1983.

15. Press, W.; Teukolsky, S.A. Savitzky-Golay Smoothing Filters. Comput. Phys. 1990, 4, 669. [CrossRef]

16. Peatross, J.; Ware, M.J. Physics of Light and Optics (Black \& White); Brigham Young University, Department of Physics: Provo, UT, USA, 2015.

17. Kull, H. Back Matter. In Laserphysik; Oldenbourg Wissenschaftsverlag: Munich, Germany, 2011. [CrossRef]

18. Giancoli, D.C. Physics for Scientists \& Engineers with Modern Physics; Pearson: London, UK, 2013.

19. Stewart, G. Laser and Fiber Optic Gas Absorption Spectroscopy; Cambridge University Press: Cambridge, UK, 2021. [CrossRef]

20. McGregor, C.; Nimmo, J.; Stothers, W. Fundamentals of University Mathematics; Elsevier: Amsterdam, The Netherlands, 2010. [CrossRef]

21. Booth, G.; Brodie, D. As Physics Study Guide; HarperCollins Publishers Limited: London, UK, 2008.

22. Zeller, W.; Naehle, L.; Fuchs, P.; Gerschuetz, F.; Hildebrandt, L.; Koeth, J. DFB Lasers Between $760 \mathrm{~nm}$ and 16 m for Sensing Applications. Sensors 2010, 10, 2492-2510. [CrossRef] [PubMed]

23. Viveiros, D.; Ribeiro, J.; Flores, D.; Ferreira, J.; Frazão, O.; Santos, J.L.; Baptista, J.M. Gas sensing using wavelength modulation spectroscopy. In Proceedings of the Second International Conference on Applications of Optics and Photonics, Aveiro, Portugal, 26-30 May 2014. 